\title{
Análise epidemiológica de doenças respiratórias entre 2015 a 2020 no território
}

\section{brasileiro}

\author{
Epidemiological analysis of respiratory diseases between 2015 to 2020 in brazilian territoy \\ Análisis epidemiológico de las enfermedades respiratorias entre 2015 y 2020 en el territorio \\ brasileño
}

Recebido: 04/06/2021 | Revisado: 09/06/2021 | Aceito: 15/06/2021 | Publicado: 29/06/2021

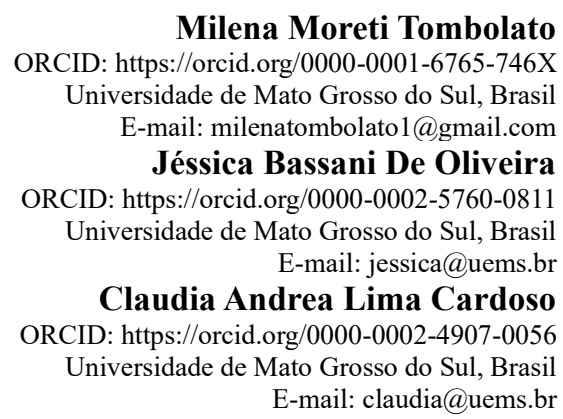

\section{Resumo}

A desigualdade social aumenta o risco de contágio doenças respiratórias. A principal preocupação da Organização Mundial da Saúde diante as doenças respiratórias são as diferentes cepas que podem ocasionar pandemias, como foi o caso do H1N1 no ano de 2009 e a COVID-19 em 2020. Nesse contexto, o objetivo deste estudo foi avaliar os percentuais de doenças respiratórias (2015-2020) comparando-as com H1N1 e COVID-19 nas regiões brasileiras. Os dados das internações e óbitos foram provenientes do Sistema de Informação Hospitalar do Sistema Único de Saúde, do Sistema de Informação sobre Mortalidade e do Ministério da Saúde, os quais foram submetidos a análise de variância (ANOVA) e de agrupamento. Os resultados apontaram que as faixas etárias mais sensíveis a desenvolver doença respiratória são crianças e idosos. Entre as regiões brasileiras a região Sul destacou-se com os maiores percentuais de internações devido a doenças respiratórias no país, enquanto nos óbitos as regiões Sul e Sudeste aproximam seus percentuais assim como as regiões Norte e Nordeste. Entretanto, comparando a mesma informação no período da pandemia COVID-19 os óbitos na região Centro-Oeste são os mais elevados. Em contrapartida, no surto de H1N1 a região Sul volta a evidenciar elevados percentuais.

Palavras-chave: Doenças respiratórias; Vírus H1N1; COVID-19.

\begin{abstract}
The Social inequality increases the risk of contagious respiratory diseases. The World Health Organization's main concern related to respiratory diseases, is the different strains of influenza that can cause pandemics, such as H1N1 in 2009 and COVID-19 in 2020. In this context, the objective of this study was to evaluate the percentages of respiratory diseases (2015-2020) comparing with H1N1 and COVID-19 in brazilian regions. Hospitalizations and deaths data came from the Hospital Information System of the Unified Health System, from the Mortality Information System and from the Ministry of Health, which were subjected to analysis of variance (ANOVA) and grouping. The results pointed out that most sensitive age groups to develop some respiratory disease, are children and elderly. Among Brazilian regions, the South region stood out with the highest percentages of hospitalizations due to respiratory diseases in the country, while in deaths, the South and Southeast regions approximate their percentages as well as the North and Northeast regions. However, comparing the same information during the COVID-19 pandemic period, deaths in the Midwest are the most significant. On the other hand, in the H1N1 outbreak, the South region once again shows high percentages.
\end{abstract}

Keywords: Respiratory tract diseases; H1N1 Virus; Coronavirus infections.

\section{Resumen}

La desigualdad social aumenta el riesgo de contagio de enfermedades respiratorias. La principal preocupación de la Organización Mundial de la Salud con respecto a las enfermedades respiratorias son las diferentes cepas que pueden causar pandemias, como fue el caso del H1N1 en 2009 y el COVID-19 en 2020. En este contexto, el objetivo de este estudio fue evaluar los porcentajes de enfermedades respiratórias (2015-2020) comparándolos con H1N1 y COVID-19 en las regiones brasileñas. Los datos de ingresos y defunciones provinieron del Sistema de Información Hospitalaria del Sistema Único de Salud, del Sistema de Información de Mortalidad y del Ministerio de Salud, los cuales fueron 
sometidos a análisis de varianza (ANOVA) y agrupamiento. Los resultados mostraron que los grupos de edad más sensibles a desarrollar enfermedades respiratorias son los niños y los ancianos. Entre las regiones brasileñas, la región Sur se destacó con los porcentajes más altos de hospitalizaciones por enfermedades respiratorias en el país, mientras que en las defunciones las regiones Sur y Sudeste aproximan sus porcentajes, así como las regiones Norte y Nordeste. Sin embargo, al comparar la misma información en el período de la pandemia de COVID-19, las muertes en la región del Medio Oeste son las más altas. Por otro lado, en el brote de H1N1, la región Sur nuevamente muestra altos porcentajes.

Palabras clave: Enfermedades respiratórias; Virus H1N1; COVID-19.

\section{Introdução}

As doenças respiratórias (DR) estão associadas principalmente com a poluição do ar, seja em queimadas de biomassa ou produzidas por veículos e indústrias, gerando um grande problema de saúde pública (Cesar et al., 2013). Fatores como baixo peso ao nascer de uma criança, falta de aleitamento materno e aglomerados de pessoas na residência, sobretudo nas regiões de periferia, aumentam a propagação das infecções respiratórias (Meneses et al., 2019).

Pires et al (2020) destaca no ano de 2013, segundo dados da Pesquisa Nacional de Saúde, entre os 20\% mais pobres da população brasileira, 94,4\% não possuem plano de saúde e a disponibilidade de leitos de Unidades de Tratamento Intensivo (UTI) são cinco vezes inferiores para usuários do Sistema Único de Saúde (SUS) do que para a parcela da população que possui acesso a rede privada.

A infecção pelo vírus influenza gera grande preocupação para a saúde pública mundial por ser facilmente transmissível e com grande capacidade mutagênica do vírus, causando uma morbimortalidade agravada entre idosos, crianças e pessoas imunodeprimidos (Departamento de Vigilância Epidemiológica, 2009). Quando comparado a outras doenças respiratórias, ela comumente leva a hospitalizações e ao óbito, além de causar o agravamento de outras doenças crônicas (Brasil, 2017).

Devido à elevada taxa de transmissão e capacidade mutagênica, as epidemias de gripe não são possíveis de prever e atingindo milhares de pessoas todos os anos. No ano de 2009 a circulação de um novo subtipo do vírus influenza A chamada "gripe suína" associada a um vírus de origem suína, H1N1(Beirigo et al., 2017) e que causou uma pandemia de repercussão social e econômica levando a Organização Mundial de Saúde (OMS) a declarar Emergência de Saúde Pública de Importância Internacional (Rossetto et al., 2016).

O ano de 2020, está marcado pela pandemia de um novo tipo de vírus proveniente da família do Coronavírus, causando uma severa síndrome respiratória aguda (SARS-CoV-2), variando em casos leves e graves, requerendo assistências hospitalar. Sabe-se que a COVID-19, nome dado à doença causada pelo vírus, possui uma alta transmissibilidade afetando pessoas de todas as nações, sua letalidade varia de acordo com faixa etária e comorbidades do indivíduo. Tal situação, fez com que o diretor da OMS declarar estado de emergência pública (Salles Neto et al., 2020).

No Brasil a ocorrência dessas doenças respiratórias é centrada principalmente nos períodos do outono e inverno (Brasil, 2017). O outono é caracterizado pelas reduções de chuvas em grande parte do País, sendo as regiões Sul, Sudeste e parte do Centro-Oeste as temperaturas ficam amenas, com as massas de ar fria e seca e temperaturas que variam de mínimas de $12^{\circ} \mathrm{C}$ a máximas de $28^{\circ} \mathrm{C}$. O inverno, nessas mesmas regiões, é a estação menos chuvosa, sendo as manhãs com maiores índices de umidade e o período da tarde os níveis ficam críticos (CPTEC, 2020).

Portanto este estudo tem como objetivo avaliar os percentuais de doenças respiratórias comparando-as com as pandemias do H1N1 e COVID-19 nas regiões brasileiras.

\section{Metodologia}

Trata-se de um estudo epidemiológico, de caráter descritivo e quantitativo, baseado em dados secundários a respeito da morbimortalidade de doenças respiratórias nas regiões do Brasil entre os períodos de 2015 a 2020 comparando-os com os anos 
de pandemia do H1N1(2009) e COVID-19 (2020). Foram utilizados dados relativos às regiões referentes às internações e óbitos por doenças respiratórias e também informações relativas a sexo e idade, provenientes do Sistema de Informação Hospitalar do Sistema Único de Saúde (SIH/SUS) e do Sistema de Informação sobre Mortalidade (SIM), disponíveis no Departamento de Informatica do SUS (DATASUS) site (http://tabnet.datasus.gov.br/cgi/deftohtm.exe?sih/cnv/niuf.def ) acessados entre 26/08/2020 a $14 / 01 / 2021$.

O SIH/SUS e SIM utilizam a 10a Revisão da Classificação Internacional de Doenças (CID 10), em que o Capítulo X, códigos J00-J99, referem-se às doenças crônicas e agudas do aparelho respiratório, e as doenças nele determinadas (infecções agudas das vias aéreas superiores, influenza [gripe] e pneumonia, outras infecções agudas das vias aéreas inferiores, outras doenças das vias aéreas superiores, doenças crônicas das vias aéreas inferiores, doenças pulmonares devidas a agentes externos, outras doenças respiratórias que afetam principalmente o interstício, afecções necróticas e supurativas das vias aéreas inferiores, outras doenças da pleura e outras doenças do aparelho respiratório. Os dados de mortalidade do H1N1 encontram-se no DATASUS separadamente dos casos das demais influenzas e do COVID-19 foram obtidos a partir do site do Ministério da Saúde no dia 14/01/2021.

As informações obtidas pela base de dados do SIH/SUS e do Ministério da Saúde foram convertidas em tabelas, transformando os valores brutos em percentuais. Posteriormente realizando-se uma análise estatística aplicando análise de variância (ANOVA) e análise de agrupamento com a construção de um dendograma. Para a realização das análises estatísticas utilizou-se a linguagem de programação R, através da ferramenta R Studio versão 1.3.959 (R Core Team). A ANOVA compara as médias de variáveis de resposta nos diferentes níveis de fator, trabalhando com conjuntos de variáveis dependentes simultaneamente (Foundation for Statistical Computing, 2021). A análise de agrupamento é uma técnica de métodos de classificação baseado na similaridade ou distância. O dendograma foi construído a partir de pares mais similares e grupos formados foram reunidos em função das similaridades decrescentes (Cohen J). Para a estratégia de agrupamento foi adotada a distância euclidiana que consiste na distância geométrica no espaço multidimensional.

\section{Resultados e Discussão}

A sociedade está sujeita a infecções das vias aéreas superiores ocasionadas pela presença de vírus no ar, provocando doenças e diversos problemas de saúde (Cruz et al., 2018). Doenças desse tipo, por serem facilmente e rapidamente transmissíveis, acarretam as epidemias e pandemias, espalhando-se por diversos países afetando grande parte da população, gerando consequências dependendo do tempo que duram (Brasil, 2019).

Existem desigualdades entre as diferentes regiões brasileiras em relação à prestação de serviços em saúde. A oferta de profissionais e serviços constituem um desafio às unidades de atendimento (Santos et al., 2020; Viacava et al., 2018). Ademais, quando há uma carência de infraestrutura domiciliar e a falta de cuidado médico adequado, o risco de contágio e propagação dessas doenças são maiores, sendo as populações que vivem nas periferias as mais atingidas (Silva Filho et al., 2017).

No Brasil, entre o período de 2015 a 2019, o número de internações hospitalares por doenças respiratórias na população foi de 5.926.687, de acordo com as informações do Sistema Único de Saúde (SUS), com uma média de 282,0/100 mil hab. Contudo, quando comparado os anos de pandemia essas taxas ficam elevadas, sendo 2009 o período do surto de H1N1, com 730,4/100 mil hab e 2020 foram 361,8/100 mil hab. Com relação à mortalidade os períodos de pandemia registraram em 2009 com 54,5/100 mil hab e 2020 com COVID-19 foram 97,4/100 mil hab, enquanto que de 2015 a 2019 foram em média 37,1/ 100 mil hab.

Nas internações, de modo geral, as doenças do aparelho respiratório acometem crianças principalmente entre a faixa etária dos 5 aos 14 anos, enquanto que nos adultos a população acima dos 50 anos são as mais atingidas. O sexo masculino é o mais afetado, representando $51 \%$ dos casos, enquanto as pessoas do sexo feminino representam $47 \%$. Entretanto, $52 \%$ dos óbitos 
são homens na faixa etária acima dos 70 anos afetados pelas doenças respiratórias e nos casos de COVID-19 acomete-se, de preferência, a partir dos 60 anos, diferindo nos casos infantis onde a COVID-19 tornar-se inexpressiva.

De acordo com Kelvin et al (2020) bebês e crianças geralmente possuem alto risco de infecções por vírus no trato respiratório devido a sua imaturidade do sistema imunológico. Enquanto Dias et al (2020) justifica os altos percentuais dos idosos por conta da diminuição das funções pulmonares e devido à idade avançada consequentemente surgem as comorbidades acentuando o risco de um avanço mais grave das doenças respiratórias. No caso das crianças em relação a COVID-19 elas apresentam uma menor suscetibilidade a doença devido a uma resposta imune inata mais ativa assim como uma respiração mais saudável (Tezer et al., 2020) Além de que, há alguns estudos que sugerem que a proteína Spike da SARS-CoV-2 liga-se à enzima angiotensina (ACE) 258, e esta em crianças é menos madura dando uma proteção a doença (Ludvigsson, 2020).

A elevada incidência de internações no sexo masculino pode fundamentar-se por fatores culturais onde a população masculina tende a ter uma menor procura dos serviços de saúde, assim como possuir hábitos pouco saudáveis (Furtado et al., 2011). Além do que se sabe que no cromossomo $X$ há uma alta variabilidade de genes relacionados ao sistema imunológico fazendo com que mulheres disponham de respostas imunes inatas e adaptativas mais fortes do que dos homens (Gemmati et al., 2020). Também em análises de dados genômicos observou-se que o sistema imunológico feminino produz uma resposta mais regulada, enquanto que os homens possuem uma expressão genética semelhante aos idosos (USP, 2020).

Ao comparar os percentuais de internações com os de óbitos, dos períodos analisados, foi possível observar uma diferença significativa entre eles $(\mathrm{p}<0,05, \mathrm{~F}=7.875)$ por meio do teste ANOVA. No que diz respeito às internações, por doenças respiratórias (DR), as regiões destaques pelo maior e menor percentual dos anos de 2015 a 2019 foram, respectivamente as regiões Sul e Sudeste. Essas mesmas regiões também são destaques no período de 2009, as quais correspondem a um percentual de $1,08 \%$ região Sul $(0,043 \%$ corresponde à influenza) e $0,599 \%$ na região Sudeste $(0,014 \%$ à influenza). Enquanto no ano de 2020 se destaca a região Norte com o menor percentual e o Sul permanecendo com os maiores valores (Tabela 1). No Brasil, de acordo com o Ministério da Saúde, foram confirmados 27.850 casos de suína, dos quais 1.632 evoluíram a óbito, representando 18,6\% das mortes mundiais e 27,7\% no continente americano (Ministério da Saúde, 2009). Em 2020, pela Agência Brasil, foram registrados 193.800 casos de óbito a atribuídos a pandemia COVID-19 (Agencia Brasil, 2020). 
Tabela 1 - Percentual de internações e óbitos em regiões e estados por doenças respiratórias (DR), H1N1 e COVID-19.

\begin{tabular}{|c|c|c|c|c|c|}
\hline$\overline{\text { Anos }}$ & & & 2015 a 2019 & & 20 \\
\hline Doença & H1N1 & $D R$ & $D R \pm D P^{*}$ & $D R$ & Covid-1 \\
\hline & & $\operatorname{Reg} i$ & & & \\
\hline Óbitos & & & & & \\
\hline Região & 0,00004 & 0,032 & $0,012 \pm 0,004$ & 0,027 & 0,110 \\
\hline Rondônia & - & 0,032 & $0,047 \pm 0,003$ & 0,028 & 0,111 \\
\hline Acre & - & 0,038 & $0,061 \pm 0,008$ & 0,031 & 0,093 \\
\hline Amazonas & - & 0,026 & $0,035 \pm 0,001$ & 0,024 & 0,146 \\
\hline Roraima & - & 0,018 & $0,033 \pm 0,005$ & 0,040 & 0,128 \\
\hline Pará & 0,00007 & 0,031 & $0,048 \pm 0,004$ & 0,024 & 0,085 \\
\hline Amapá & - & 0,020 & $0,038 \pm 0,007$ & 0,015 & 0,116 \\
\hline Tocantins & - & 0,029 & $0,039 \pm 0,005$ & 0,025 & 0,082 \\
\hline
\end{tabular}

\section{Internações}

$\begin{array}{lcccc}\text { Região } & - & \mathbf{0 , 8 3} & 0,147 \pm 0,025 & \mathbf{0 , 3 1} \\ \text { Rondônia } & - & 0,82 & 0,752 \pm 0,033 & 0,31 \\ \text { Acre } & - & 0,59 & 0,413 \pm 0,031 & 0,21 \\ \text { Amazonas } & - & 0,41 & 0,379 \pm 0,021 & 0,21 \\ \text { Roraima } & - & 0,49 & 0,534 \pm 0,083 & 0,33 \\ \text { Pará } & - & 0,99 & 0,690 \pm 0,028 & 0,36 \\ \text { Amapá } & - & 0,40 & 0,447 \pm 0,088 & 0,23 \\ \text { Tocantins } & - & 0,84 & 0,523 \pm 0,060 & 0,25\end{array}$

Região Nordeste

\section{Óbitos}

$\begin{array}{lccccc}\text { Região } & \mathbf{0 , 0 0 0 0 4} & \mathbf{0 , 0 4 2} & 0,016 \pm 0,003 & \mathbf{0 , 0 3 0} & \mathbf{0 , 0 8 8} \\ \text { Maranhão } & - & 0,022 & 0,042 \pm 0,003 & 0,021 & 0,065 \\ \text { Piauí } & - & 0,038 & 0,066 \pm 0,007 & 0,029 & 0,090 \\ \text { Ceará } & - & 0,048 & 0,077 \pm 0,007 & 0,038 & 0,111 \\ \text { Rio Grande do Norte } & - & 0,043 & 0,063 \pm 0,005 & 0,024 & 0,090 \\ \text { Paraíba } & 0,00010 & 0,045 & 0,077 \pm 0,004 & 0,034 & 0,096 \\ \text { Pernambuco } & 0,00004 & 0,057 & 0,083 \pm 0,004 & 0,029 & 0,104 \\ \text { Alagoas } & - & 0,041 & 0,059 \pm 0,003 & 0,028 & 0,078\end{array}$


Research, Society and Development, v. 10, n. 7, e46610716819, 2021

(CC BY 4.0) | ISSN 2525-3409 | DOI: http://dx.doi.org/10.33448/rsd-v10i7.16819

$\begin{array}{ccccc}- & 0,038 & 0,054 \pm 0,004 & 0,031 & 0,114 \\ 0,00009 & 0,035 & 0,050 \pm 0,004 & 0,029 & 0,064\end{array}$

Sergipe

Bahia

Internações

Região

Maranhão

Piauí

Ceará

Rio Grande do Norte

Paraíba

Pernambuco

Alagoas

Sergipe

Bahia

Óbitos

Região

Paraná

Santa Catarina

Rio Grande do Sul

Internações

Região
Paraná
Santa Catarina
Rio Grande do Sul

0,77

0,72

1,14

0,70

0,62

0,95

0,52

0,71

0,30

0,92

Região Sul

$\begin{array}{ll}0,134 \pm 0,030 & \mathbf{0 , 2 7} \\ 0,660 \pm 0,073 & 0,37 \\ 0,761 \pm 0,030 & 0,43 \\ 0,537 \pm 0,051 & 0,29 \\ 0,337 \pm 0,019 & 0,19 \\ 0,531 \pm 0,024 & 0,24 \\ 0,461 \pm 0,028 & 0,24 \\ 0,401 \pm 0,019 & 0,20 \\ 0,319 \pm 0,032 & 0,19 \\ 0,574 \pm 0,048 & 0,27\end{array}$

, 27

$\begin{array}{lllll}\mathbf{0 , 0 0 1 8 1} & \mathbf{0 , 0 6 9} & 0,021 \pm 0,004 & \mathbf{0 , 0 5 0} & \mathbf{0 , 0 8 3} \\ 0,00154 & 0,060 & 0,078 \pm 0,003 & 0,045 & 0,077 \\ 0,00072 & 0,049 & 0,066 \pm 0,004 & 0,044 & 0,080 \\ 0,00259 & 0,082 & 0,093 \pm 0,005 & 0,055 & 0,086\end{array}$

$\begin{array}{lll}\mathbf{1 , 0 8} & 0,216 \pm 0,021 & \mathbf{0 , 4 0 6} \\ 1,17 & 0,849 \pm 0,014 & 0,425 \\ 0,82 & 0,755 \pm 0,027 & 0,368 \\ 1,05 & 0,867 \pm 0,049 & 0,385\end{array}$

Sudeste

Óbitos

Região
Minas Gerais
Espírito Santo
Rio de Janeiro
São Paulo

$\begin{array}{lllll}\mathbf{0 , 0 0 0 3 8} & \mathbf{0 , 0 6 9} & 0,023 \pm 0,002 & \mathbf{0 , 0 4 4} & \mathbf{0 , 1 1 0} \\ 0,00041 & 0,056 & 0,081 \pm 0,003 & 0,039 & 0,061 \\ 0,00012 & 0,045 & 0,061 \pm 0,006 & 0,039 & 0,134 \\ 0,00051 & 0,077 & 0,098 \pm 0,003 & 0,041 & 0,155 \\ 0,00030 & 0,068 & 0,088 \pm 0,002 & 0,045 & 0,106\end{array}$


Research, Society and Development, v. 10, n. 7, e46610716819, 2021

(CC BY 4.0) | ISSN 2525-3409 | DOI: http://dx.doi.org/10.33448/rsd-v10i7.16819

$\begin{array}{lcccc}\text { Região } & - & \mathbf{0 , 5 9 9} & 0,128 \pm 0,004 & \mathbf{0 , 3 1 1} \\ \text { Minas Gerais } & - & 0,693 & 0,594 \pm 0,013 & 0,345 \\ \text { Espírito Santo } & - & 0,549 & 0,552 \pm 0,014 & 0,328 \\ \text { Rio de Janeiro } & - & 0,452 & 0,303 \pm 0,020 & 0,202 \\ \text { São Paulo } & - & 0,565 & 0,504 \pm 0,007 & 0,311\end{array}$

\section{Centro-Oeste}

\section{Óbitos}

$\begin{array}{lccccc}\text { Região } & \mathbf{0 , 0 0 0 6 3} & \mathbf{0 , 0 4 7} & 0,016 \pm 0,003 & \mathbf{0 , 0 3 3} & \mathbf{0 , 1 2} \\ \text { Mato Grosso do Sul } & 0,00072 & 0,051 & 0,075 \pm 0,006 & 0,050 & 0,09 \\ \text { Mato Grosso } & 0,00037 & 0,039 & 0,052 \pm 0,003 & 0,031 & 0,13 \\ \text { Goiás } & 0,00083 & 0,047 & 0,067 \pm 0,003 & 0,028 & 0,10 \\ \text { Distrito Federal } & 0,00017 & 0,036 & 0,044 \pm 0,003 & 0,021 & 0,15\end{array}$

\section{Internações}

\begin{tabular}{lccccc} 
Região & - & $\mathbf{0 , 8 8 9}$ & $0,150 \pm 0,022$ & $\mathbf{0 , 3 2 9}$ & - \\
Mato Grosso do Sul & - & 0,837 & $0,711 \pm 0,031$ & 0,394 & - \\
Mato Grosso & - & 0,873 & $0,563 \pm 0,016$ & 0,282 & - \\
Goiás & - & 0,900 & $0,532 \pm 0,051$ & 0,277 & - \\
Distrito Federal & - & 0,605 & $0,528 \pm 0,074$ & 0,347 & - \\
\hline
\end{tabular}

* DP: Desvio padrão *DR: Doenças Respiratórias Fonte: Autores.

$\mathrm{Na}$ análise do dendograma (Figura 1) foi possível verificar a formação de dois grandes grupos (internações e óbitos), ressaltando as particularidades, percebido anteriormente pelo teste ANOVA. O grupo de internações se divide formando dois subgrupos: o primeiro formado pelas regiões Centro-Oeste, Sudeste, Nordeste e Norte e, o segundo subgrupo formado pela região Sul. Este distanciamento da região Sul das demais regiões ocorre por apresentar um valor elevado nas internações por doenças respiratórias em 2009. No grupo dos óbitos formaram-se, também, outros dois subgrupos Sul e Sudeste e, o segundo subgrupo composto pelas regiões Nordeste, Norte e Centro-Oeste. As regiões Nordeste, Norte e Centro-Oeste apresentam características semelhantes, porém ocorre ainda uma segunda subdivisão, formada pelas regiões Nordeste e Norte. Estas regiões apresentam uma menor distância euclidiana entre óbitos por H1N1 em 2009. As regiões Sul e Sudeste se assemelham nos percentuais de internações e óbitos por doenças respiratórias no ano de 2009 e óbitos entre os anos de 2015 a 2020. 
Figura 1 - Agrupamento em similaridades por internações e óbitos das regiões brasileiras.

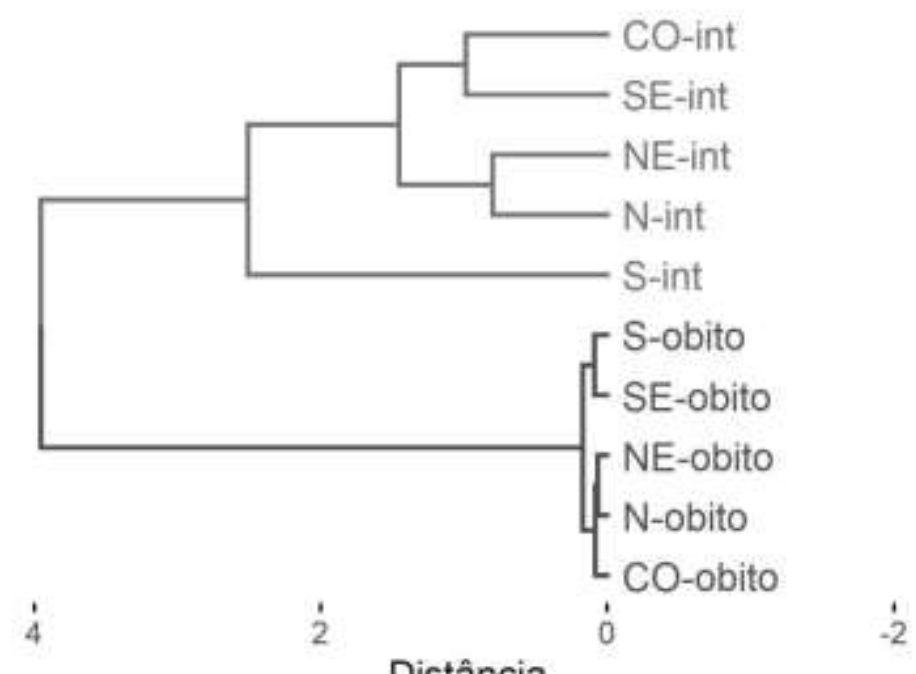

*CO- Centro-Oeste; SE- Sudeste; NE- Nordeste; N-Norte; S-Sul

* Int- Internações

Fonte: Autores.

A análise realizada entre todos os anos, no quesito dos óbitos, constatou uma diferença significativa entre os percentuais nas regiões do país ( $\mathrm{p}<0,05, \mathrm{~F}=41.05$ ). No ano de 2020 ao comparar os casos por doenças respiratórias e COVID-19 observouse uma diferença significativa entre elas $(\mathrm{p}<0,05, \mathrm{~F}=58.12)$. A região mais atingida por doenças respiratórias foi à região Sul, enquanto a menos atingida foi a Norte, mas quando comparadas com os óbitos devido a COVID-19, a região Centro-Oeste foi a mais atingida, possuindo um percentual de 0,12\%. Enquanto os períodos de 2015 a 2019 a região com maior percentual de obtidos por DR foi o Sudeste e essa mesma região sobressaiu no ano de 2009 com 0,069\%, entretanto em relação a H1N1 a região Centro-Oeste foi evidenciada o percentual mais elevado (Tabela 1).

Nas regiões com elevado desenvolvimento industrial e econômico há uma maior exposição aos poluentes em razão à grande quantidade de fábricas e ao elevado número de veículos (Torres et al., 2020). Os centros urbanos são os mais afetados pela poluição e além de sofrerem com a saúde da população há um impacto negativo na perspectiva econômica e social, isso porque ocorre um aumento nos custos dos sistemas de saúde e a população mais carente são vistas como exemplos dos problemas causados pela contaminação (Beirigo et al, 2017).

Uma nova infecção por um novo coronavírus foi descrito no final de 2019 na China causando uma síndrome respiratória aguda grave a SARS-CoV-2, sendo o agente patogênico da COVID-19, o qual atinge gravemente o sistema respiratório humano e devido ao rápido e alto contágio entre as pessoas, há um elevado número de casos e óbitos (Zhu et al., 2020)

No ano de 2020 a região Sul assumiu os maiores percentuais de internações e óbitos, seguido do Sudeste posto que estas regiões sejam mais populosas do Brasil pode-se relacionar com a pandemia da COVID-19. Segundo Oliveira et al (2020) no Sul observou-se um aumento nos óbitos por pneumonia na região, sendo um sintoma do SARS-CoV-2, e o Sudeste responsável por $80 \%$ do espalhamento dos casos iniciais no país.

Na região Sul, o Paraná sobressai diante dos outros estados ao que se refere às internações por doenças respiratórias durante os anos de 2015 a 2019 possuindo um percentual elevado.Entretanto, ao comparar esse percentual com o ano de 2009 um período de pandemia do H1N1, o estado dispara e chega a 1,17\%, sendo de que desse percentual 0,039\% refere-se a influenza. Em 2020, na pandemia da COVID-19, esse percentual de doenças respiratórias volta a diminuir, porém o estado do Paraná 
continua em destaque dentre os demais estados do Sul. Em contrapartida, o Rio Grande do Sul assume a posição do estado com os maiores percentuais de óbitos, principalmente durante as pandemias do H1N1 e COVID-19 chegando em 2,59^10 $0^{-3} \%$ e $0,086 \%$ respectivamente. E em relação às doenças respiratórias, dentre todos os períodos analisados, 2009 possui o maior percentual dos óbitos (Tabela 1).

Nas internações, o estado de Minas Gerais, na região Sudeste destaca-se com os maiores percentuais de doenças respiratórias (DR) principalmente no ano de 2009 em que esse percentual chega a 0,69\% sendo que $0,011 \%$ refere-se a influenza e em 2020 ele sofre uma diminuição, ainda sim permanecendo como o estado em maior evidência, sobretudo entre os períodos de 2015 a 2019. Todavia quando comparado com os óbitos por DR o Rio de Janeiro é o que mais se sobressai, principalmente no período de 2009 , onde os percentuais chegam a $0,077 \%$, sendo o percentual de $5,1 \times 10^{-4} \%$ representando os casos de H1N1, em contrapartida em 2020 esse percentual por doenças respiratórias decai, não sendo mais o Rio de Janeiro o destaque da região Sudeste. Contudo, ao comparar com os óbitos por COVID-19, o estado volta a se sobressair (Tabela 1).

O Mato Grosso do Sul destacou-se entre os estados da região Centro-Oeste por seus percentuais de internações por doenças respiratórias serem mais elevados. Sendo destaque nos anos de 2015 a 2019 com 0,18\% e em 2020 com 0,39\%, diferindo do ano de 2009 no qual o estado não se sobressaiu na região tanto pelas DR quanto pelos casos de influenza (Tabela 1). Ao que corresponde aos óbitos o mesmo estado é destaque nas doenças respiratórias principalmente nos anos de 2009 e 2020, entretanto nas doenças do H1N1, ele se encontra como o segundo maior e na COVID-19 é o de menor percentual dentre todos os estados (Tabela 1).

Estudos sobre pandemias, como o H1N1, demonstraram que a desigualdade social é um fator determinante para uma maior taxa de transmissão assim como para o agravamento dessas doenças infecciosas (Tricco et al., 2012). Segundo Borges et al (2020) indivíduos menos escolarizados possuem maior incidência para os fatores de comorbidades de risco para a COVID19. Isso reflete na população das idades mais avançadas, resultando em uma chance de $47 \%$ de pessoas com nível educacional mais baixo de pertencer ao grupo de risco do que aqueles que possuem nível superior.

\section{Conclusão}

A realização deste estudo possibilitou a identificação das regiões brasileiras mais atingidas pelas internações e óbitos por consequência de doenças que acometem o sistema respiratório, bem como às faixas etárias e o sexo que foram mais acometidos com essas doenças. As doenças respiratórias foram comparadas às epidemias do H1N1 no ano de 2009 e à COVID19 no ano de 2020. Observou-se uma diferença significativa, por meio do teste ANOVA, entre os casos por doenças respiratórias e COVID-19 no ano de 2020.

Os casos de doenças respiratórias de forma geral no ano de 2020 diminuíram em comparação aos demais anos analisados neste estudo. Em 2020, houve também as primeiras notificações de casos de COVID-19 no país. Deste modo, para trabalhos futuros, sugere-se ampliar o estudo comparativo entre casos de COVID-19 em relação às doenças respiratórias nos municípios que compõem as regiões.

\section{Agradecimentos}

Ao suporte da Fundação de Apoio ao Desenvolvimento do Ensino, Ciência e Tecnologia do Estado de Mato Grosso do Sul (FUNDECT) (número de concessão 71/700.139/2018; 036/20108 e SIAFEM 028991) e Conselho Nacional de Desenvolvimento Científico e Tecnológico (CNPq) para CALC (número de concessão 311975/2018-6) e bolsa do Programa Institucional de Iniciação Científica (PIBIC-UEMS). 


\section{Referências}

Agência Brasil. EBC (2020). COVID-19: Brasil tem 193,9 mil mortes e 7,61 milhões de casos. <https://agenciabrasil.ebc.com.br/saude/noticia/2020-12/covid19-brasil-tem-1938-mil-mortes-e-761-milhoes-de-casos>.

Beirigo, A. P. T., da Silva Pereira, I., \& Costa, P. S. (2017). Influenza A (H1N1): revisão bibliográfica. SaBios-Revista de Saúde e Biologia, 12 (2), 53-67.

Borges, G. M., \& Crespo, C. D. (2020). Aspectos demográficos e socioeconômicos dos adultos brasileiros e a COVID-19: uma análise dos grupos de risco a partir da Pesquisa Nacional de Saúde, 2013. Cadernos de Saúde Pública, 36, e00141020.

Brasil. Ministério da Saúde (2019). Informações técnicas e recomendações sobre a sazonalidade de influenza 2019. Secretaria de Vigilância em Saúde. Brasília: Ministério da Saúde, 6p. https://portalarquivos2.saude.gov.br/images/pdf/2019/marco/20/Plano-de-Conting--ncia-para-Sazonalidade-e-Epidemias-de-Influenza--Final-enviado-19.03.2019.pdf.

Brasil. Ministério da Saúde. Secretaria de Vigilância em Saúde (2017). Coordenação-Geral de Desenvolvimento da Epidemiologia em Serviços. Guia Vigilancia em Saúde: volume único. Ministério da Saúde. p. 9-17. https://portalarquivos2.saude.gov.br/images/PDF/2017/outubro/16/Volume-Unico-2017.pdf.

Cesar, A. C. G.; et al (2013). Association between exposure to particulate matter and hospital admissions for respiratory disease in children. Revista de saúde pública, 47, 1209-1212.

Clima Estados- CPTEC/INPE. Inpe.br. http://clima1.cptec.inpe.br/estacoes/pt.

Cohen, J. Statistical Power Analysis for the Behavioral Sciences. (2nd ed.) L. Erlbaum

Cruz, G. M. A. D., Lima, R. C. D., Costa, D. D. O., \& Bastianini, L. F. M. (2017). H1N1 víurs: perfil epidemiológico do vírus no período da pandemia de 2009 e 2010 nas cinco regiões brasileiras. <https://core.ac.uk/download/pdf/335080276.pdf>.

Departamento de Vigilância Epidemiológica, Secretaria de Vigilância em Saúde, Ministério da Saúde (2009). Influenza. In: Departamento de Vigilância Epidemiológica, Secretaria de Vigilância em Saúde, Ministério da Saúde, organizador. Guia de vigilância epidemiológica. 7a Ed. Brasília: Ministério da Saúde. p. 1-23. https://bvsms.saude.gov.br/bvs/publicacoes/guia_vigilancia_epidemiologica_7ed.pdf.

Dias, F. L. T., Mendonça, F. D., Pinto, G. M., Borges, I. S. C., \& de Oliveira, S. V. (2020). Doenças respiratórias no Triângulo Mineiro: Análise epidemiológica e projetiva com a pandemia de COVID-19. Journal of Health \& Biological Sciences, 8(1), 1-6.

Estudo sugere que as mulheres têm melhor resposta imune à covid-19 (2020). Jornal da USP. https://jornal.usp.br/ciencias/estudo-sugere-que-as-mulheres-temmelhor-resposta-imune-a-covid-19.

Foundation for Statistical Computing. Vienna. Austria. Published 2021. https://www.r-project.org/

Furtado, M. A., da Silva, R. H., Zaia, J. E., \& Nascif-Júnior, I. A. (2011). Influência da vacinação contra influenza em idosos na epidemiologia da hospitalização por pneumonia. Investigação, 11(2).

Gemmati, D., Bramanti, B., Serino, M. L., Secchiero, P., Zauli, G., \& Tisato, V. (2020). COVID-19 and individual genetic susceptibility/receptivity: role of ACE1/ACE2 genes, immunity, inflammation and coagulation. might the double X-chromosome in females be protective against SARS-CoV-2 compared to the single X-chromosome in males?. International journal of molecular sciences, 21(10), 3474.

Kelvin, A. A., \& Halperin, S. (2020). COVID-19 in children: the link in the transmission chain. The Lancet Infectious Diseases, $20(6), 633-634$.

Ludvigsson, J. F. (2020). Systematic review of COVID-19 in children shows milder cases and a better prognosis than adults. Acta paediatrica, 109(6), 10881095 .

Menezes, R. A. D. M., Pavanitto, D. R., \& Nascimento, L. F. C. (2019). Exposição a poluentes do ar e doença respiratória em meninos e meninas. Revista Paulista de Pediatria, 37(2), 166-172.

Oliveira, G. L., Ribeiro, A. P., Pereira, C. C. A., \& Machado, C. J. (2020). O novo coronavírus e a pneumonia: análise comparativa de internações e óbitos no Brasil entre 2019 e 2020. Revista Thema, 18, 332-342.

Pires, L. N., Carvalho, L., \& XAVIER, L. D. L. (2020). COVID-19 e desigualdade: a distribuição dos fatores de risco no Brasil. Experiment Findings, 21.

R Core Team. R: A Language and Environment for Statistical Computing. R

Rossetto, E. V., \& Luna, E. J. D. A. (2016). Relacionamento entre bases de dados para vigilância da pandemia de influenza A (H1N1) pdm09, Brasil, 20092010. Cadernos de Saúde Pública, 32, e00014115.

Salles Neto, L. L. D., Martins, C. B., Chaves, A. A., Konstantyner, T. C. R. D. O., Yanasse, H. H., Campos, C. B. L. D., ... \& Soares, F. D. S. (2020). Forecast UTI: aplicativo para previsão de leitos de unidades de terapia intensiva no contexto da pandemia de COVID-19. Epidemiologia e Serviços de Saúde, 29, e2020391.

Santos, A. C., Góes, F. G. B., Pereira, F. M. V., Camilo, L. A., Bonifácio, M. C. S., \& Knupp, V. M. D. A. O. (2020). Perfil clínico epidemiológico de crianças admitidas em unidade pediátrica. Revista Enfermagem UERJ, 28, 46533.

Silva Filho, E. B. D., Silva, A. L. D., Santos, A. D. O. D., Dall'acqua, D. S. V., \& Souza, L. F. B. (2017). Infecções respiratórias de importância clínica: uma revisão sistemática.

Tezer, H., \& Demirdağ, T. B. (2020). Novel coronavirus disease (COVID-19) in children. Turkish journal of medical sciences, 50 (SI-1), 592-603. 
Research, Society and Development, v. 10, n. 7, e46610716819, 2021

(CC BY 4.0) | ISSN 2525-3409 | DOI: http://dx.doi.org/10.33448/rsd-v10i7.16819

Torres, L. M., da Silva Pinheiro, C. D. P., Azevedo, S. D., Rodrigues, P. R. S., \& Sandim, D. P. R. (2020). Poluição atmosférica em cidades brasileiras: uma breve revisão dos impactos na saúde pública e meio ambiente. Naturae, 2(1), 23-33.

Tricco, A. C., Lillie, E., Soobiah, C., Perrier, L., \& Straus, S. E. (2012). Impact of H1N1 on socially disadvantaged populations: systematic review. PLoS One, 7(6), e39437.

Viacava, F., Oliveira, R. A. D. D., Carvalho, C. D. C., Laguardia, J., \& Bellido, J. G. (2018). SUS: oferta, acesso e utilização de serviços de saúde nos últimos 30 anos. Ciência \& saúde coletiva, 23, 1751-1762.

Zhu, Y., Xie, J., Huang, F., \& Cao, L. (2020). Association between short-term exposure to air pollution and COVID-19 infection: Evidence from China. Science of the total environment, 727,138704 